\title{
New design of grab mechanism in plastic glove machine
}

\author{
Wei-Chun Hsu, Ming-Jin Jiang ${ }^{a}$ and Gui-Liang Liu \\ Graduate School of OptoMechatronics and Materials, WuFeng University, Taiwan
}

\begin{abstract}
The aim of this study is to design a new type of grab mechanism for plastic bags machine in order to tear apart the plastic gloves from the plastic bags and stack them into a pile automatically. A grab mechanism with four bar linkage is developed and the main part of the grab mechanism is the clip which will pick up the plastic gloves and drag them to the other side. After a distance of drag, the clip will drop the plastic gloves to the collecting plate. The clips are fixed on the connecting rod of the four-bar linkage and the path of the clip can be traced by the position analysis of the four bar linkage. The path of the clip can be tracked by determination of the four links' lengths. This path tracking will be one of the advantages of this new mechanism because it provides the different drag distance to grab difference size of plastic gloves by simply adjusting the lengths of four links.
\end{abstract}

\section{Preface}

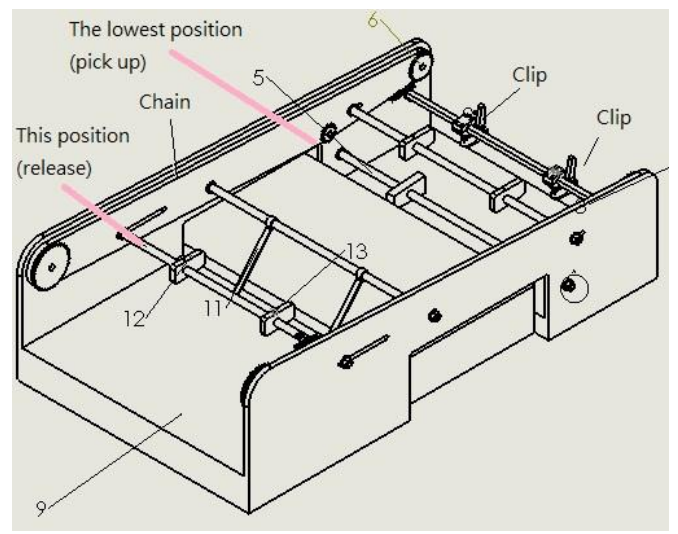

Figure 1. Chain conveyor belt mechanism.

In order to achieve the needs of customers and the globalization of the market considerations, the traditional machinery must develop more competitive products. In view of the medical use, the plastic gloves are usually made of PE and EVA materials. During the conventional production process, the plastic gloves are torn off the plastic bags by worker's hand and this is not good for medical use. In order to solve this problem, an automatic release mechanism should be invented. The general release

\footnotetext{
${ }^{a}$ Corresponding author : ingmar.hsu@wfu.edu.tw

(C) The Authors, published by EDP Sciences. This is an open access article distributed under the terms of the Creative Commons Attribution License 4.0 (http://creativecommons.org/licenses/by/4.0/).
} 
mechanism [1] is composed of chain conveyor belt which connects the clips to tear off the plastic gloves. During the required process, the clip goes downward to pick up the plastic gloves from the plastic bags which have been cut with shapes of gloves. The clip drags the glove forward and upward for a distance and then the clip will drop the plastic gloves to the collecting plate as shown in figure 1 . According to the design requirements of this path, we design a new grab mechanism. A new grab mechanism is designed by a four-bar linkage $[2,3]$ which provides high rigidity characteristics and is better than the low rigidity of chain.

\section{New design of grab mechanism}

A new grab mechanism design [4] is composed of a four-bar linkage and clips. The two clips are fixed in the connecting rod of the four-bar linkage as shown in figure 2. In this way, the path of the clip looks like the path of the coupling rod in the four-bar linkage. In order to determine the path of the clip, it can be obtained by means of the four-bar linkage position analysis. According to the design requirements of the clip path, the relationship between the length ratio of each link and the path of the clip is analyzed. The path length is adjusted by the link length to achieve the requirement of the production process.

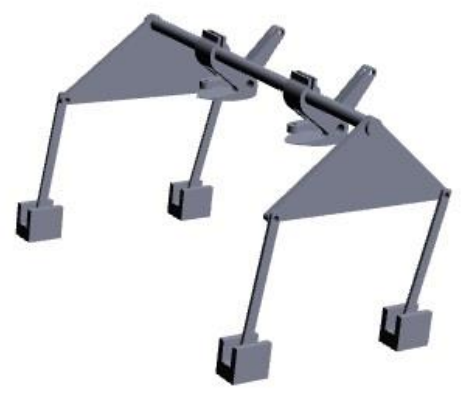

Figure 2. 3D diagram of grab mechanism.

\section{Path analysis of clips}

Before the size of the total mechanism is determined, the shape of the clip path should be determined in accordance with the requirement. Figure 3 shows a typical vector drawing of four-bar linkage. The vectors $\boldsymbol{r}_{\mathbf{1}}, \boldsymbol{r}_{\mathbf{2}}, \boldsymbol{r}_{\mathbf{3}}$ and $\boldsymbol{r}_{\mathbf{4}}$ represent the fixed link, drive link, connecting link and output link, respectively. The clip locates on point A of the connecting link. The sum of these vectors can be expressed as follow $[5,6]$ :

$$
r_{2}+r_{3}-r_{1}-r_{4}=0
$$

Thus

$$
\begin{aligned}
& r_{2} \sin \theta_{2}+r_{3} \sin \theta_{3}-r_{1} \sin \theta_{1}-r_{4} \sin \theta_{4}=0 \\
& r_{2} \cos \theta_{2}+r_{3} \cos \theta_{3}-r_{l} \cos \theta_{1}-r_{4} \cos \theta_{4}=0
\end{aligned}
$$

The lengths, $r_{1}, r_{2}, r_{3}$ and $r_{4}$, of the mechanism, position angle $\theta_{1}$ and input angle $\theta_{2}$ are given. There are only two unknowns, $\theta_{3}$ and $\theta_{4}$, in equation 2 and equation 3 . Because there are trigonometric functions in two equations, these need application of iterative method to solve the problem $[4,6]$ to obtain the answers of $\theta_{3}$ and $\theta_{4}$. 


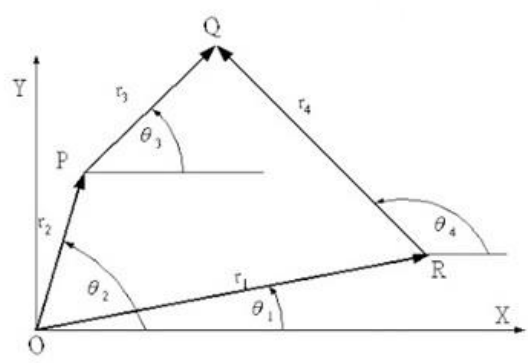

(a) Vector Loop for four-bar linkage

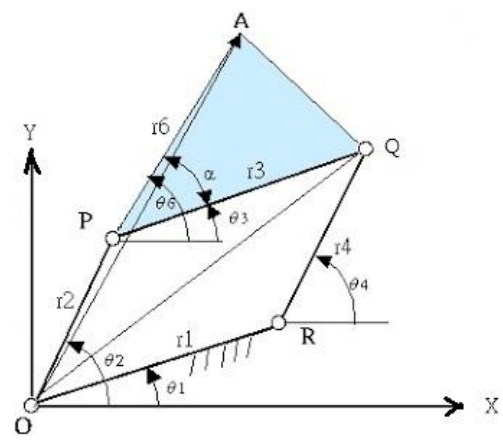

(b) Vector for the couplar link

Figure 3. Vectors for the four-bar linkage.

After the angles of $\theta_{3}$ and $\theta_{4}$ are obtained, the horizontal and vertical components of the position vector $r_{A}$ of point $\mathrm{A}$ are determined.

$$
\begin{aligned}
& r_{A x}=r_{2} \cos \theta_{2}+r_{6} \cos \theta_{6} \\
& r_{A y}=r_{2} \sin \theta_{2}+r_{6} \sin \theta_{6}
\end{aligned}
$$

Where $\theta_{6}=\theta_{3}+\theta$.Thus the position of the clip can be obtained. The loop position of the clip can be calculated at each angle of $\theta_{2}$ when angle $\theta_{2}$ varies from 0 to $360^{\circ}$. In this way the loop position of the clip can be displayed by connecting the calculated positions when angle $\theta_{2}$ varies from $0^{\circ}$ to $360^{\circ}$. Therefore different path will be obtained according to the different size of the grab mechanism and this will be a good advantage to achive the production requirement of different glove sizes.

\section{Results and discussion}

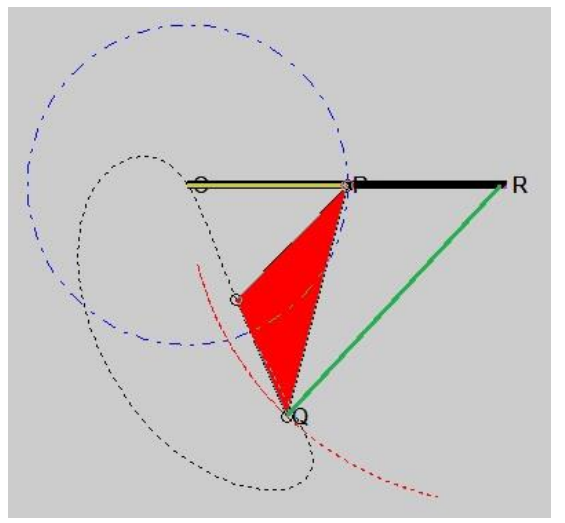

Figure 4. Trajectories for the four-bar linkage with length ratio $4: 2: 3: 4: 2\left(\theta_{1}=0^{\circ}\right)$.

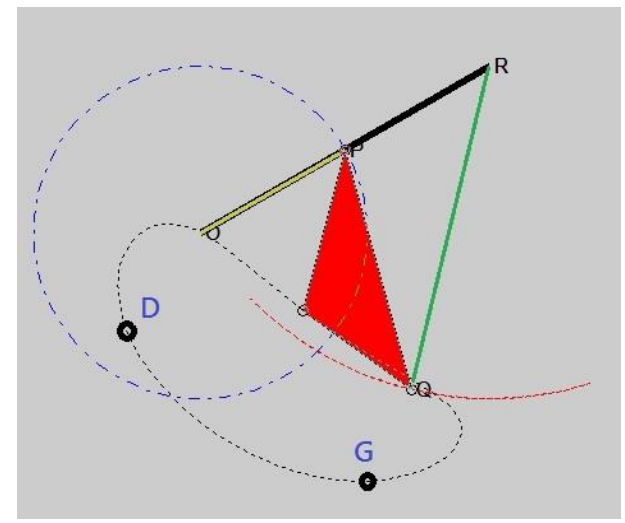

Figure 5. Trajectories for the four-bar linkage with length ratio 4: $2: 3: 4: 2\left(\theta_{1}=45^{\circ}\right)$.

According to the mechanism design theory and automatic technique, a grab mechanism with four bar linkage is developed. The clip fixed on the mechanism goes downward to pick up the plastic gloves from the plastic bags which have been cut with shapes of gloves. The clip drags the glove forward and upward for a distance and then the clip will drop the plastic gloves to the collecting plate and then return to its original position to pick up another one. The shape of this clip path is determined with the size of the four-bar linkage. According to the kinematic trajectory analysis of the plastic glove release mechanism [4], it can be known that the path shape of the clip can be adjusted by changing the lengths ratio of the four-bar linkage. Therefore, the four rods 1, 2, 3, 4 and the length of the coupling rod is set 
to be $4: 2: 3: 4: 2$. The drive link 2 is driven directly by the motor rotation, so link 2 needs to turn $360^{\circ}$. The angle is $30^{\circ}$ and the angle $\theta_{1}$ of the fixed link 1 is 0 degrees in this case. Figure 4 shows the blue, red and black trajectoriers represents the pathes of point $\mathrm{P}$ on link 2, point $\mathrm{Q}$ on link 4 and the clip, respectively. The black dotted trajectory for the clip path does not achieve the requirement of the movement of picking up the plastic gloves. This time the angle $\theta_{1}$ of the fixed link 1 is switched to 45 degrees in this case. And the diagram is shown in figure 5.

This diagram shows a better trajectory for the clip. The clip can pick up the plastic glove on point $\mathrm{G}$ and drop it on point $\mathrm{D}$. For a better choice of the clip trajectory, the clip pick up and drag the plastic glove left and upward from point $\mathrm{G}$ to point $\mathrm{D}$ and this route is the working process. The clip returns to point $\mathrm{G}$ to grab another glove after drop the last one and this route is the non-working process. In order to reduce the cycling time of the clip, the length of the trajectory should be reduced. After many times of trials, a better choice of length ratio for the four rods 1, 2, 3, 4 and the length of the coupling rod is set to be 4.5: $2: 3: 4: 3$. And the angle $\theta_{1}$ of the fixed link 1 is also 45 degrees in this case. The result is shown in figure 6 .

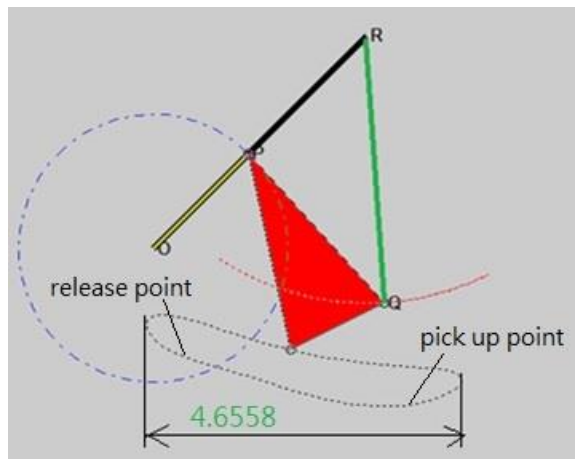

Figure 6. Trajectories for the four-bar linkage with length ratio $4.5: 2: 3: 4: 3\left(\theta_{1}=45^{\circ}\right)$

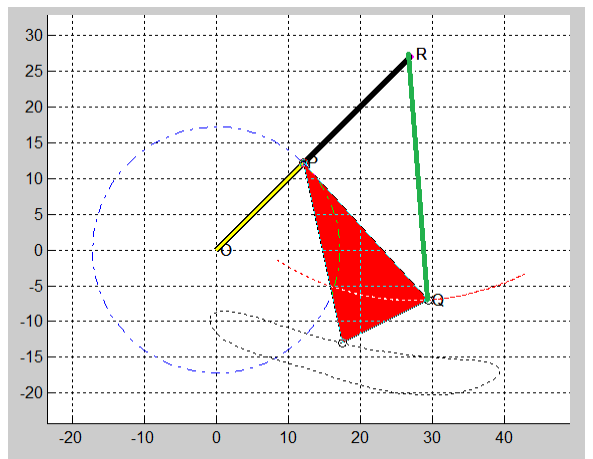

Figure 7. The design trajectories as request

The width of the trajectory in X-axis direction can be obtained by calculate the difference of minimal and maximal position of the clip. In this case, the horizontal width is 4.6558 as shown in figure 6 . If the width is required to be $40 \mathrm{~cm}$, then the lengths of the $\operatorname{rod} 1, \operatorname{rod} 2, \operatorname{rod} 3, \operatorname{rod} 4$ and coupler are $38.66 \mathrm{~cm}, 17.18 \mathrm{~cm}, 25.77 \mathrm{~cm}, 34.37 \mathrm{~cm}$ and $25.77 \mathrm{~cm}$, respectively. The result is shown in figure 7. It can be seen from the diagram, the grab mechanism requires working space about $60 \mathrm{~cm}$ wide and $50 \mathrm{~cm}$ high. In this way we can design the grab mechanism with many different link lengths to serve grapping requirement of different glove lengths.

The purpose of this study is to design a new grab mechanism of a plastic product to replace the conventional machine. The basic four-bar position analysis can be used to calculate the clip path quickly under different design requirements. In the direction of the adjustment, the width of the clip path can be obtained by the program analysis. By means of the scale enlargement, the actual size of the new grab mechanism can be determined.

\section{Conclusion}

This study shows that a new design of grab mechanism in automatic plastic bag machine can be carried out. The development of a new design can provide high production capacity and high rigidity. The number of clips on the connecting link can be increase to provide more production capacity. The mechine can be easily set up for different glove length production by adjusting the length of the four links. The development of technology can make the machine more competitive in Taiwan, Europe and the United States markets. 


\section{References}

1. M.T. Ho, Wu-Feng University Technical Reports, Design of Molding Mechanism of PE Plastic Glove Machine, 2-5 (2015)

2. C.C. Tsai, Thesis of Department of Mechanical and Automation Engineering National Kaohsiung First University of Science and Technology, The Optimal Dimensional Synthesis of Four-Bar Linkage for Open Path Generation, Kaohsiung, Taiwan ROC (2012)

3. Y.H. Su, Thesis of Department of Mechanical Engineering Kun Shan University, Design, Analysis, and Fabrication of a Composite Four-Bar Linkage for Pick and Place, Tainan, Taiwan ROC (2012)

4. S.Y. Zong, Thesis of Graduate School of Opto-Mechatronics and Meterials Wu-Feng University, Design of Grab Mechanism of Plastic Glove Machine (2016)

5. Y.H. Kang, Mechanism (Gau Lih Book Co., Ltd., 1996)

6. Y.H. Fan, Y.K. Zeng and Z.S. Chen, Mechanism (Gau Lih Book Co., Ltd., 2014) 